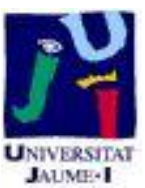

Título artículo / Títol article: On the fundamental tone of minimal submanifolds with controlled extrinsic curvature
Autores / Autors
Gimeno, Vicente

Revista:

Potential Analysis, 2013, published online 07 June

Versión / Versió:

Cita bibliográfica / Cita bibliogràfica (ISO 690):
Post-print de l'autor

GIMENO, Vicent. On the fundamental tone of minimal submanifolds with controlled extrinsic curvature. Potential Analysis, 2013, p. 1-12. 


\title{
ON THE FUNDAMENTAL TONE OF MINIMAL SUBMANIFOLDS WITH CONTROLLED EXTRINSIC CURVATURE
}

\author{
VICENT GIMENO
}

\begin{abstract}
The aim of this paper is to obtain the fundamental tone for minimal submanifolds of the Euclidean or hyperbolic space under certain restrictions on the extrinsic curvature. We show some sufficient conditions on the norm of the second fundamental form that allow us to obtain the same upper and lower bound for the fundamental tone of minimal submanifolds in a Cartan-Hadamard ambient manifold. As an intrinsic result, we obtain a sufficient condition on the volume growth of a Cartan-Hadamard manifold to achieve the lowest bound for the fundamental tone given by McKean.
\end{abstract}

\section{INTRODUCTION}

Let $(M, g)$ be a Riemannian manifold, the fundamental tone $\lambda^{*}(M)$ of $M$ is defined by

$$
\lambda^{*}(M)=\inf \left\{\frac{\int_{M}|\nabla f|^{2}}{\int_{M} f^{2}} ; f \in L_{1,0}^{2}(M) \backslash\{0\}\right\}
$$

where $L_{1,0}^{2}(M)$ is the completion of $C_{0}^{\infty}(M)$ with respect to the norm $\|\varphi\|^{2}=$ $\int_{M} \varphi^{2}+\int_{M}|\nabla \varphi|^{2}$.

The fundamental tone is a powerful tool in the challenging area of geometric analysis, being a classic feature its relation with the curvature of the manifold. We refer to the book of I. Chavel [ $[$ ] for a discussion of these and related concepts.

For non-positively curved manifolds, lower bounds for the fundamental tone are well-known. For these cases, McKean gave the following bounds for the fundamental tone of a Cartan-Hadamard manifold.

Theorem 1.1 (See [14]). Let $M^{n}$ be a complete, simply connected manifold with sectional curvature bounded above by $\kappa<0$. Then

$$
\lambda^{*}(M) \geq \frac{-(n-1)^{2} \kappa}{4} .
$$

Similary, L.P. Cheung and L.F. Leung showed that minimal submanifolds of the hyperbolic space retain this lower bound of the ambient manifold.

Theorem 1.2 (See [8]). Let $M^{n}$ be a complete minimal submanifold in the hyperbolic space $\mathbb{H}^{m}(\kappa)$ of constant sectional curvature $\kappa<0$. Then

$$
\lambda^{*}(M) \geq \frac{-(n-1)^{2} \kappa}{4} .
$$

Observe that inequality (1.2) and inequality (1.3) are sharp in the sense that when $M$ is the hyperbolic space $\mathbb{H}^{n}(\kappa)$ (considered as a manifold or considered as a totally geodesic submanifold of $\left.\mathbb{H}^{m}(\kappa)\right)$ the fundamental tone $\lambda^{*}(M)$ satisfies $\lambda^{*}(M)=\frac{-(n-1)^{2} \kappa}{4}($ see [5] or [14]).

Moreover, it is known that other minimal submanifolds exist as well as the hyperbolic space with that lowest fundamental tone. For example, all the classical 
minimal catenoids in the hyperbolic space $\mathbb{H}^{3}(-1)$ given by $\mathrm{M}$. do Carmo and $\mathrm{M}$. Dajczer in [4] achieve equality in (1.3) as shown by A. Candel.

Theorem 1.3 (See [3]). The fundamental tone of the minimal catenoids (given in 4. ) in the hyperbolic space $\mathbb{H}^{3}(-1)$ is

$$
\lambda^{*}(M)=\frac{1}{4}
$$

The purpose of this paper is to provide geometric conditions under which a submanifold (or a manifold) attains the equality in the inequality (1.3) (or inequality (1.2), respectively). Let us emphasize that the spherical catenoids given in the previous example, as it was analyzed in [17, have finite $L^{2}$-norm of the second fundamental form (denoted by $A$ throughout this paper)

$$
\int_{M}|A|^{2} d \mu<\infty
$$

This could suggest that the finiteness of the $L^{2}$-norm of the second fundamental form for minimal surfaces in the hyperbolic space implies the lowest fundamental tone. This is in fact true, and is the statement of our first theorem.

Theorem A. Let $M^{2}$ be a minimal surface immersed in the hyperbolic space $\mathbb{H}^{m}(\kappa)$ of constant sectional curvature $\kappa<0$. Suppose that $M^{2}$ has finite total extrinsic curvature, i.e, $\int_{M}|A|^{2} d \mu<\infty$. Then, the fundamental tone satisfies

$$
\lambda^{*}(M)=\frac{-\kappa}{4} .
$$

Our approach to the problem makes use of our previous results about the influence of the extrinsic curvature restrictions on the volume growth of the submanifold (see [11, [10, [12] or \$2), and also the relation between the finite volume growth and the fundamental tone as a new tool (see the Main Theorem).

For higher dimensional minimal submanifolds of the hyperbolic space, under an appropriate decay of the norm of the second fundamental form on the extrinsic distance (see $\$ 2$ for a precise definition) we obtain

Theorem B. Let $M^{n}$ be a minimal submanifold properly immersed in the hyperbolic space $\mathbb{H}^{m}(\kappa)$ of constant sectional curvature $\kappa<0$. Suppose moreover that $n>2$ and the submanifold is of faster than exponential decay of its extrinsic curvature. Namely, there exists a point $p \in M$ such that

$$
|A|_{x} \leq \frac{\delta\left(r_{p}(x)\right)}{e^{2 \sqrt{-\kappa} r_{p}(x)}}
$$

where $\delta(r)$ is a function such that $\delta(r) \rightarrow 0$ when $r \rightarrow \infty$ and $r_{p}$ is the extrinsic distance function. Then, the fundamental tone satisfies

$$
\lambda^{*}(M)=\frac{-(n-1)^{2} \kappa}{4} .
$$

We also prove for minimal submanifolds of the Euclidean space the following well known result (see for example [2])

Theorem C. Let $M^{n}$ be a minimal submanifold immersed in the Euclidean space $\mathbb{R}^{m}$ with finite total scalar curvature, i.e, $\int_{M}|A|^{n} d \mu<\infty$. Then

$$
\lambda^{*}(M)=0 \text {. }
$$

Remark a. As far as we know, the question 1.5 given in [2] about the existence (or not) of complete minimal submanifolds properly immersed in the $\mathbb{R}^{m}$ with positive fundamental tone is still an open question. In any case, if such kind of immersion 
exists, from our Main Theorem the immersion should be of infinite volume growth, and from Theorem $\mathrm{E}$ the submanifold has no an extrinsic doubling property.

Finally, as an intrinsic version of our Main Theorem one may obtain the following theorem in the direction of the McKean's Theorem 1.1

Theorem D. Let $M^{n}$ be a complete, simply connected manifold with sectional curvature bounded from above by $\kappa<0$. Suppose moreover that there exists a point $p \in M$ such that

$$
\sup _{R>0} \frac{\operatorname{Vol}\left(B_{R}^{M}(p)\right)}{\operatorname{Vol}\left(B_{R}^{\kappa}\right)}<\infty \quad,
$$

where $B_{R}^{M}(p)$ is the geodesic ball in $M$ centered at $p$ of radius $R$, and $B_{R}^{\kappa}$ is the geodesic ball in $\mathbb{H}^{n}(\kappa)$ of the same radius $R$. Then

$$
\lambda^{*}(M)=\frac{-(n-1)^{2} \kappa}{4} .
$$

Remark b. In view of the intrinsic version of the results of [9] for complete, simply connected, 2-dimensional manifold $M^{2}$ with Gaussian curvature $K_{M}$ bounded from above by $K_{M} \leq \kappa<0$, the condition (1.8) can be achieved provided the following

$$
\int_{M}\left(\kappa-K_{M}\right) d \mu<\infty
$$

In particular (in view of [9]), for every 2-dimensional Cartan-Hadamard manifold $M$ which is asymptotically locally $\kappa$-hyperbolic of order 2 (see [18, 9]) and with sectional curvatures bounded from above by $K_{M} \leq \kappa<0$, the fundamental tone satisfies

$$
\lambda^{*}(M)=\frac{-\kappa}{4}
$$

The proof of the three previous extrinsic theorems (Theorem A, Theorem B and Theorem C) uses the fact that under the hypothesis of the theorems the submanifold has finite volume growth $\left(\lim _{R \rightarrow \infty} \mathcal{Q}(R)<\infty\right.$, see \$2 and $\$ 2.1$ for precise definitions and see also [19] for an alternative definition in terms of projective volume). Hence, the theorems will be proved using the following Main Theorem:

Main Theorem. Let $M^{n}$ be a n-dimensional minimal submanifold properly immersed in a simply connected Cartan-Hadamard manifold $N$ of sectional curvature $K_{N}$ bounded from above by $K_{N} \leq \kappa \leq 0$. Suppose that

$$
\lim _{R \rightarrow \infty} \mathcal{Q}(R)<\infty \text {. }
$$

Then,

$$
\lambda^{*}(M)=-\frac{(m-1)^{2} \kappa}{4}
$$

To obtain the Main Theorem we estimate upper bounds for the fundamental tone. According to S.T. Yau (20]) it is important to find upper bounds for the fundamental tone. In the case of stable minimal submanifolds of the hyperbolic space, A. Candel gave in 2007 the following upper bound for the fundamental tone

Theorem 1.4 (See [3]). Let $M$ be a complete simply connected stable minimal surface in the 3 -dimensional hyperbolic space $\mathbb{H}^{3}(-1)$. Then the fundamental tone of $M$ satisfies

$$
\frac{1}{4} \leq \lambda^{*}(M) \leq \frac{3}{4}
$$


Using only as hypothesis the finiteness of the $L^{2}$-norm of the second fundamental form, K. Seo has recently generalized the result of Theorem 1.4 without using the hypothesis about the simply connectedness

Theorem 1.5 (See 17). Let $M^{n}$ be a complete stable minimal hypersurface in $\mathbb{H}^{n+1}(-1)$ with $\int_{M}|A|^{2} d \mu<\infty$. Then we have

$$
\frac{(n-1)^{2}}{4} \leq \lambda^{*}(M) \leq n^{2}
$$

Another achievement of this paper is that using only the finiteness of the $L^{2}$-norm of the second fundamental form (Theorem A), or an appropriate decay of the norm of the second fundamental form, we can also remove the hypothesis about the stability, and obtain not only an inequality but an equality on the fundamental tone.

Remark c. Note that the finiteness of the $L^{2}$-norm of the second fundamental form of a minimal surface in the hyperbolic space does not imply the stability of the surface. In fact, M. do Carmo and M. Dajczer also proved in 4 that there exist some unstable spherical catenoids in $\mathbb{H}^{3}(-1)$. And observe, moreover, that the codimension of the submanifold plays no role in our theorems, in contrast to what happens in the theorems from A. Candel and K. Seo where the codimension must be 1 .

The structure of the paper is as follows.

In $₫ 2$ we recall the definition of the extrinsic distance function, the extrinsic ball and the volume growth function $\mathcal{Q}$. Showing that under the hypothesis of Theorems $\mathrm{A}, \mathrm{B}$, and $\mathrm{C}$ the submanifold has finite volume growth

$$
\sup _{R>0} \mathcal{Q}(R)<\infty \quad \text {. }
$$

In 93 we will prove the Main Theorem. Finally as a corollary from the proof of the Main Theorem, supposing that the submanifold has an extrinsic doubling property we state the Theorem E where is obtained lower and upper bounds for the fundamental tone of a minimal submanifold properly immersed in a CartanHadamard ambient manifold.

\section{Preliminaries}

The proof of Theorems A, B and C is based on upper and lower bounds for the fundamental tone. The lower bounds are well known (see Theorem 1.2), but to obtain upper bounds we use the so called volume growth function and its relation to the behavior of the extrinsic curvature. The volume growth function is the quotient between the volume of an extrinsic ball of radius $R$ and the geodesic ball of the same radius $R$ in an appropriate real space form.

Let $\mathbb{K}^{n}(\kappa)$ denote the $n$-dimensional simply connected real space form of constant sectional curvature $\kappa \leq 0$, recall that the volume of the geodesic sphere $S_{R}^{\kappa}$ and the geodesic ball $B_{R}^{\kappa}$ of radius $R$ in $\mathbb{K}^{n}(\kappa)$ are (see [13])

$$
\operatorname{Vol}\left(S_{R}^{\kappa}\right)=\omega_{n} \mathrm{~S}_{\kappa}(R)^{n-1} \quad \operatorname{Vol}\left(B_{R}^{\kappa}\right)=\int_{0}^{R} \operatorname{Vol}\left(S_{s}^{\kappa}\right) d s \quad,
$$

being $\omega_{n}$ the volume of the geodesic sphere of radius 1 in $\mathbb{R}^{n}$, where $\mathrm{S}_{\kappa}$ is the usual function

$$
\mathrm{S}_{\kappa}(t)=\left\{\begin{array}{l}
t \quad \text { if } \kappa=0, \\
\frac{\sinh (\sqrt{-\kappa} t)}{\sqrt{-\kappa}} \quad \text { if } \quad \kappa<0 .
\end{array}\right.
$$

And the mean curvature pointing inward of the geodesic spheres of radius $R$ is 


$$
\mathrm{Ct}_{\kappa}(t)=\frac{\mathrm{S}_{\kappa}^{\prime}(t)}{\mathrm{S}_{\kappa}(t)}
$$

On the other hand, given a submanifold $M$ immersed in a simply connected Cartan-Hadamard manifold $N$ of sectional curvature $K_{N}$ bounded from a above by $K_{N} \leq \kappa \leq 0$, the extrinsic ball $M_{p}^{R}$ centered at $p \in M$ of radius $R$ is the sublevel set of the extrinsic distance function $r_{p}$, where the extrinsic distance function is

Definition 2.1 (Extrinsic distance function). Let $\varphi: M \rightarrow N$ be an immersion from the manifold $M$ to the simply connected Cartan-Hadamard manifold of sectional curvature $K_{N}$ bounded from above by $K_{N} \leq \kappa \leq 0$. Given a point $p \in M$, the extrinsic distance function $r_{p}: M \rightarrow \mathbb{R}^{+}$is defined by

$$
r_{p}(x)=\operatorname{dist}^{N}(\varphi(p), \varphi(x)),
$$

where dist ${ }^{N}$ denotes the usual geodesic distance function in $N$.

Therefore the extrinsic ball $M_{p}^{R}$ centered at $p \in M$ of radius $R$ is

$$
M_{p}^{R}:=\left\{x \in M: r_{p}(x)<R\right\} .
$$

With the extrinsic ball and the geodesic ball we can define the volume growth function

Definition 2.2 (Volume growth function). Let $\varphi: M^{n} \rightarrow N$ be an immersion from the $n$-dimensional manifold $M$ to the simply connected Cartan-Hadamard manifold of sectional curvature $K_{N}$ bounded from above by $K_{N} \leq \kappa \leq 0$. The volume growth function $\mathcal{Q}: \mathbb{R}^{+} \rightarrow \mathbb{R}^{+}$is given by

$$
\mathcal{Q}(R):=\frac{\operatorname{Vol}\left(M_{p}^{R}\right)}{\operatorname{Vol}\left(B_{R}^{\kappa}\right)} .
$$

Where $\operatorname{Vol}\left(B_{R}^{\kappa}\right)$ is the geodesic ball of radius $R$ in $\mathbb{K}^{n}(\kappa)$.

2.1. Volume growth of minimal submanifolds of controlled extrinsic curvature. Firstly to prove the Theorems A, B and $\mathrm{C}$ we have to study the behavior of the volume comparison function for minimal submanifolds of a Cartan-Hadamard manifold. Let us recall the following monotonicity formula

Proposition 2.3 (Monotonicity formula, see [15]). Let $\varphi: M^{n} \rightarrow N$ be an immersion from the $n$-dimensional manifold $M$ to the simply connected CartanHadamard manifold of sectional curvature $K_{N}$ bounded from above by $K_{N} \leq \kappa \leq 0$. Then, the volume growth function is a non-decreasing function.

Secondly to prove Theorems A, B, and C we need to check that an appropriate control of their second fundamental form allow us to apply the Main Theorem (\$3). In order to apply the Main Theorem the submanifold should have finite volume growth

$$
\lim _{R \rightarrow \infty} \mathcal{Q}(R)<\infty \quad .
$$

But we can apply the Main Theorem under the assumptions of the Theorems A, $\mathrm{B}$ and $\mathrm{C}$ because of the following three theorems that we recall here:

Theorem 2.4 (See [1, [6] and [11]). Let $M^{n}$ be a minimal submanifold immersed in the Euclidean space $\mathbb{R}^{m}$. If $M^{n}$ has finite total scalar curvature

Then

$$
\int_{M}|A|^{n} d \mu<\infty
$$

$$
\sup _{R>0} \mathcal{Q}(R)<\infty .
$$


Theorem 2.5 (See [7, [1] and [16]). Let $M^{2}$ be a minimal surface immersed in the hyperbolic space $\mathbb{H}^{m}(\kappa)$ of constant sectional curvature $\kappa<0$ or in the Euclidean space $\mathbb{R}^{m}$. If $M$ has finite total extrinsic curvature, namely $\int_{M}|A|^{2} d \mu<\infty$, then $M$ has finite topological type, and

$$
\sup _{R>0} \mathcal{Q}(R) \leq \frac{1}{4} \int_{M}|A|^{2} d \mu+\chi(M),
$$

being $\chi(M)$ the Euler characteristic of $M$.

And

Theorem 2.6 (see [12]). Let $M^{n}$ be a minimal $n$-dimensional submanifold properly immersed in the hyperbolic space $\mathbb{H}^{m}(\kappa)$ of constant sectional curvature $\kappa<0$. If $n>2$ and the submanifold is of faster than exponential decay of its extrinsic curvature, namely, there exists a point $p \in M$ such that

$$
|A|_{x} \leq \frac{\delta\left(r_{p}(x)\right)}{e^{2 \sqrt{-\kappa} r_{p}(x)}}
$$

where $\delta(r)$ is a function such that $\delta(r) \rightarrow 0$ when $r \rightarrow \infty$. Then the submanifold has finite topological type, and

$$
\sup _{R>0} \mathcal{Q}(R) \leq \mathcal{E}(M)
$$

being $\mathcal{E}(M)$ the (finite) number of ends of $M$.

\section{Proof of the Main Theorem: volume growth Behavior and FUNDAMENTAL TONE}

The way to proof the equality (1.12) in the Main Theorem is to obtain the same upper and lower bound for the fundamental tone. Hence, first of all, we need lower bounds for the fundamental tone. But the lower bounds are well known, and it is straight forward in a similar way to Theorem 1.2 that

$$
\lambda^{*}(M) \geq \frac{-(n-1)^{2} \kappa}{4} .
$$

The above lower bound can also be proved using the Cheeger isoperimetric constant of the submanifold. Taking into account that the Cheeger constant $h(M)$ of a minimal submanifold $M^{n}$ properly immersed in a Cartan-Hadamard manifold $N$ of sectional curvatures $K_{N}$ bounded from above by $K_{N} \leq \kappa \leq 0$ is (see [10])

$$
h(M) \geq(n-1) \sqrt{-\kappa},
$$

therefore,

$$
\lambda^{*}(M) \geq \frac{h(M)^{2}}{4} \geq \frac{-(n-1)^{2} \kappa}{4} .
$$

To obtain the upper bounds for the fundamental tone, we use the Rayleigh quotient definition (1.1) with an appropriate testing function. The first step to obtain the testing function is to define the real function $\phi: \mathbb{R} \rightarrow \mathbb{R}$, given by

$$
\phi(t)=\left\{\begin{array}{l}
f(t) \quad \text { if } t \in\left[\frac{R}{2}, R\right], \\
0 \quad \text { otherwise }
\end{array}\right.
$$

where the function $f: \mathbb{R} \rightarrow \mathbb{R}$ is

$$
f(s)=\frac{\sin \left(\frac{2 \pi\left(s-\frac{R}{2}\right)}{R}\right)}{\operatorname{Vol}\left(S_{s}^{\kappa}\right)^{1 / 2}} .
$$


Now, the second step to take to construct the testing function $\Phi$, is to transplant $\phi$ to $M$ using the extrinsic distance function by the following definition:

$$
\Phi: M \rightarrow \mathbb{R} ; \quad \Phi(x)=\phi\left(r_{p}(x)\right)
$$

By the Rayleigh quotient definition and the coarea formula

$$
\begin{aligned}
\lambda^{*}(M) & \leq \frac{\int_{M}\langle\nabla \Phi, \nabla \Phi\rangle d \mu}{\int_{M} \Phi^{2} d \mu}=\frac{\int_{M}\left(\phi^{\prime}\right)^{2}\left\langle\nabla r_{p}, \nabla r_{p}\right\rangle d \mu}{\int_{M} \phi^{2} d \mu} \leq \frac{\int_{M}\left(\phi^{\prime}\right)^{2} d \mu}{\int_{M} \phi^{2} d \mu} \\
& =\frac{\int_{0}^{R}\left[\int_{\partial M_{p}^{s}} \frac{\left(\phi^{\prime}\right)^{2}}{|\nabla r|}\right] d s}{\int_{0}^{R}\left[\int_{\partial M_{p}^{s}} \frac{\phi^{2}}{|\nabla r|}\right] d s}=\frac{\int_{\frac{R}{2}}^{R}\left(\phi^{\prime}(s)\right)^{2}\left[\int_{\partial M_{p}^{s}} \frac{1}{|\nabla r|}\right] d s}{\int_{\frac{R}{2}}^{R} \phi^{2}(s)\left[\int_{\partial M_{p}^{s}} \frac{1}{|\nabla r|}\right] d s} \\
& =\frac{\int_{\frac{R}{2}}^{R}\left(\phi^{\prime}(s)\right)^{2}\left(\operatorname{Vol}\left(M_{p}^{s}\right)\right)^{\prime} d s}{\int_{\frac{R}{2}}^{R} \phi^{2}(s)\left(\operatorname{Vol}\left(M_{p}^{s}\right)\right)^{\prime} d s} .
\end{aligned}
$$

Using the following two lemmas

\section{Lemma 3.1.}

$$
\mathcal{Q}(s) \operatorname{Vol}\left(S_{s}^{\kappa}\right) \leq\left(\operatorname{Vol}\left(M_{p}^{s}\right)\right)^{\prime}=(\ln \mathcal{Q}(s))^{\prime} \operatorname{Vol}\left(B_{s}^{\kappa}\right) \mathcal{Q}(s)+\mathcal{Q}(s) \operatorname{Vol}\left(S_{s}^{\kappa}\right)
$$

Proof. From the definition of $\mathcal{Q}$ and taking into account that $\mathcal{Q}$ is a non-decreasing function (by proposition 2.3)

$$
(\ln \mathcal{Q}(s))^{\prime}=\frac{\left(\operatorname{Vol} M_{p}^{s}\right)^{\prime}}{\left(\operatorname{Vol} M_{p}^{s}\right)}-\frac{\operatorname{Vol}\left(S_{s}^{\kappa}\right)}{\operatorname{Vol}\left(B_{s}^{\kappa}\right)} \geq 0 .
$$

So,

$$
\mathcal{Q}(s) \operatorname{Vol}\left(S_{s}^{\kappa}\right) \leq\left(\operatorname{Vol}\left(M_{p}^{s}\right)\right)^{\prime}=(\ln \mathcal{Q}(s))^{\prime} \operatorname{Vol}\left(B_{s}^{\kappa}\right) \mathcal{Q}(s)+\mathcal{Q}(s) \operatorname{Vol}\left(S_{s}^{\kappa}\right)
$$

Lemma 3.2. There exists an upper bound function $\Lambda: \mathbb{R}^{+} \rightarrow \mathbb{R}^{+}$such that:

$$
\frac{\int_{0}^{R}\left(\phi^{\prime}\right)^{2} \operatorname{Vol}\left(S_{s}^{\kappa}\right) d s}{\int_{0}^{R} \phi^{2} \operatorname{Vol}\left(S_{s}^{\kappa}\right) d s} \leq \Lambda(R),
$$

and

$$
\lim _{R \rightarrow \infty} \Lambda(R)=\frac{-(m-1) \kappa}{4}
$$

Proof.

$$
\frac{\int_{0}^{R}\left(\phi^{\prime}(s)\right)^{2} \operatorname{Vol}\left(S_{s}^{\kappa}\right) d s}{\int_{0}^{R} \phi(s)^{2} \operatorname{Vol}\left(S_{s}^{\kappa}\right) d s}=\frac{\int_{\frac{R}{2}}^{R}\left(f^{\prime}(s)\right)^{2} \operatorname{Vol}\left(S_{s}^{\kappa}\right) d s}{\int_{\frac{R}{2}}^{R} f(s)^{2} \operatorname{Vol}\left(S_{s}^{\kappa}\right) d s}
$$

But

$$
\begin{aligned}
\left(f^{\prime}(s)\right)^{2} & =\frac{\left(-\frac{m-1}{2} \mathrm{Ct}_{\kappa}(s) \sin \left(\frac{2 \pi\left(s-\frac{R}{2}\right)}{R}\right)+\frac{2 \pi}{R} \cos \left(\frac{2 \pi\left(s-\frac{R}{2}\right)}{R}\right)\right)^{2}}{\operatorname{Vol}\left(S_{s}^{\kappa}\right)} \\
& \leq \frac{\frac{(m-1)^{2}}{4} \mathrm{Ct}_{\kappa}(s)^{2} \sin \left(\frac{2 \pi\left(s-\frac{R}{2}\right)}{R}\right)^{2}+\frac{4 \pi^{2}}{R^{2}}+\frac{2(m-1) \pi}{R} \mathrm{Ct}_{\kappa}(s)}{\operatorname{Vol}\left(S_{s}^{\kappa}\right)}
\end{aligned}
$$


And, since $\mathrm{Ct}_{\kappa}(t)$ is a non-increasing function and $\int_{\frac{R}{2}}^{R} \sin \left(\frac{2 \pi\left(s-\frac{R}{2}\right)}{R}\right)^{2} d s=\frac{R}{4}$

$$
\begin{aligned}
\frac{\int_{0}^{R}\left(\phi^{\prime}(s)\right)^{2} \operatorname{Vol}\left(S_{s}^{\kappa}\right) d s}{\int_{0}^{R} \phi(s)^{2} \operatorname{Vol}\left(S_{s}^{\kappa}\right) d s} \leq & \frac{(m-1)^{2}}{4} \mathrm{Ct}_{\kappa}(R / 2)^{2}+\frac{8 \pi^{2}}{R^{2}} \\
& +\frac{4 \pi(m-1)}{R} \mathrm{Ct}_{\kappa}(R / 2)
\end{aligned}
$$

Then, letting

$$
\Lambda(R):=\frac{(m-1)^{2}}{4} \mathrm{Ct}_{\kappa}(R / 2)^{2}+\frac{8 \pi^{2}}{R^{2}}+\frac{4 \pi(m-1)}{R} \mathrm{Ct}_{\kappa}(R / 2)
$$

and taking into account that

$$
\lim _{R \rightarrow \infty} \mathrm{Ct}_{\kappa}(R / 2)=\sqrt{-\kappa}
$$

the lemma is proven.

Denoting now, $F(R):=\left(\frac{(m-1)^{2}}{4} \mathrm{Ct}_{\kappa}(R / 2)^{2}+\frac{4 \pi^{2}}{R^{2}}+\frac{2(m-1) \pi}{R} \mathrm{Ct}_{\kappa}(R / 2)\right)$ and $\delta(R):=$ $\int_{\frac{R}{2}}^{R}(\ln \mathcal{Q}(s))^{\prime} d s$, applying the lemma 3.1 and the lemma 3.2 to inequality (3.5) we get since $\frac{\operatorname{Vol}\left(B_{s}^{\kappa}\right)}{\operatorname{Vol}\left(S_{s}^{\kappa}\right)}$ is a non-decreasing function

$$
\begin{aligned}
\lambda^{*}(M) & \leq \frac{\mathcal{Q}(R)}{\mathcal{Q}\left(\frac{R}{2}\right)} \frac{\int_{\frac{R}{2}}^{R}\left(\phi^{\prime}(s)\right)^{2}(\ln \mathcal{Q}(s))^{\prime} \operatorname{Vol}\left(B_{s}^{\kappa}\right) d s+\int_{\frac{R}{2}}^{R}\left(\phi^{\prime}(s)\right)^{2} \operatorname{Vol}\left(S_{s}^{\kappa}\right) d s}{\int_{\frac{R}{2}}^{R} \phi^{2}(s) \operatorname{Vol}\left(S_{s}^{\kappa}\right) d s} \\
& \leq \frac{\mathcal{Q}(R)}{\mathcal{Q}\left(\frac{R}{2}\right)}\left(\frac{4}{R} \int_{\frac{R}{2}}^{R}\left(\phi^{\prime}(s)\right)^{2}(\ln \mathcal{Q}(s))^{\prime} \operatorname{Vol}\left(B_{s}^{\kappa}\right) d s+\Lambda(R)\right) \\
& \leq \frac{\mathcal{Q}(R)}{\mathcal{Q}\left(\frac{R}{2}\right)}\left[\frac{\operatorname{Vol}\left(B_{R}^{\kappa}\right)}{\operatorname{Vol}\left(S_{R}^{\kappa}\right)} \frac{4}{R} F(R) \delta(R)+\Lambda(R)\right]
\end{aligned}
$$

Letting $R$ tend to infinity and taking into account that

$$
\begin{aligned}
\lim _{R \rightarrow \infty} F(R) & =-\frac{(m-1)^{2} \kappa}{4}, \\
\lim _{R \rightarrow \infty} \delta(R) & =0, \\
\lim _{R \rightarrow \infty} \frac{\operatorname{Vol}\left(B_{R}^{\kappa}\right)}{\operatorname{Vol}\left(S_{R}^{\kappa}\right)} \frac{4}{R} & =\left\{\begin{array}{l}
\frac{4}{m-1} \text { if } \kappa=0, \\
0 \text { if } b<0 .
\end{array}\right. \\
\lim _{R \rightarrow \infty} \frac{\mathcal{Q}(R)}{\mathcal{Q}\left(\frac{R}{2}\right)} & =1, \\
\lim _{R \rightarrow \infty} \Lambda(R) & =-\frac{(m-1)^{2} \kappa}{4} .
\end{aligned}
$$

we conclude the proof of the theorem.

Remark d. Observe that the finiteness of the volume growth function

$$
\lim _{R \rightarrow \infty} \mathcal{Q}(R)<\infty,
$$

is only used in the proof of the Main Theorem to achieve

$$
\lim _{R \rightarrow \infty} \frac{\mathcal{Q}(R)}{\mathcal{Q}\left(\frac{R}{2}\right)}=1
$$

Therefore, we could use the slightly weaker assumption on the volume growth given by the limit (3.18) instead the assumption on the finite volume growth of the 
submanifold (limit (3.17)), or use an extrinsic doubling property to obtain two sides estimates for the fundamental tone

Theorem E. Let $M^{n}$ be a n-dimensional minimal submanifold properly immersed in a simply connected Cartan-Hadamard manifold $N$ of sectional curvature $K_{N}$ bounded from above by $K_{N} \leq \kappa \leq 0$. Suppose that the immersion has an extrinsic doubling property, namely

$$
\frac{\mathcal{Q}(R)}{\mathcal{Q}\left(\frac{R}{2}\right)}<C
$$

Then,

$$
-\frac{(m-1)^{2} \kappa}{4} \leq \lambda^{*}(M) \leq-\frac{C(m-1)^{2} \kappa}{4}
$$

\section{REFERENCES}

[1] Anderson, M.T.: The compactification of a minimal submanifold in Euclidean space by the Gauss map (1984)

[2] Bessa, G.P., Costa, M.S.: On submanifolds with tamed second fundamental form. Glasg. Math. J. 51(3), 669-680 (2009).

[3] Candel, A.: Eigenvalue estimates for minimal surfaces in hyperbolic space. Trans. Amer. Math. Soc. 359(8), 3567-3575 (electronic) (2007). DOI 10.1090/S0002-9947-07-04104-9. URL http://dx.doi.org/10.1090/S0002-9947-07-04104-9

[4] do Carmo, M., Dajczer, M.: Rotation hypersurfaces in spaces of constant curvature. Trans. Amer. Math. Soc. 277(2), 685-709 (1983).

[5] Chavel, I.: Eigenvalues in Riemannian geometry, Pure and Applied Mathematics, vol. 115. Academic Press Inc., Orlando, FL (1984). Including a chapter by Burton Randol, With an appendix by Jozef Dodziuk

[6] Chen, Q.: On the volume growth and the topology of complete minimal submanifolds of a Euclidean space. J. Math. Sci. Univ. Tokyo 2(3), 657-669 (1995)

[7] Chern, S.s., Osserman, R.: Complete minimal surfaces in Euclidean $n$-space. J. Analyse Math. 19, 15-34 (1967)

[8] Cheung, L.F., Leung, P.F.: Eigenvalue estimates for submanifolds with bounded mean curvature in the hyperbolic space. Math. Z. 236(3), 525-530 (2001).

[9] Esteve, A., Palmer, V.: Chern-Osserman inequality for minimal surfaces in a cartanhadamard manifold with strictly negative sectional curvatures (2011). Accepted in Arkiv för Matematik.

[10] Gimeno, V., Palmer, V.: Volume growth of submanifolds and the Cheeger isoperimetric constant (2011) . To appear in Proceedings of the AMS.

[11] Gimeno, V., Palmer, V.: Extrinsic isoperimetry and compactification of minimal surfaces in Euclidean and hyperbolic spaces. Israel Journal of Mathematics 2(194), 539-553 (2013).

[12] Gimeno, V., Palmer, V.: Volume growth, number of ends, and the topology of a complete submanifold. Journal of Geometric Analysis pp. 1-22 (2012). DOI 10.1007/s12220-012-9376-3. URL http://dx.doi.org/10.1007/s12220-012-9376-3

[13] Gray, A.: Tubes, Progress in Mathematics, vol. 221, second edn. Birkhäuser Verlag, Basel (2004). With a preface by Vicente Miquel

[14] McKean, H.: An upper bound to the spectrum of $\Delta$ on a manifold of negative curvature. J. Differ. Geom. 4, 359-366 (1970)

[15] Palmer, V.: Isoperimetric inequalities for extrinsic balls in minimal submanifolds and their applications. J. London Math. Soc. (2) 60(2), 607-616 (1999).

[16] Qing, C., Yi, C.: Chern-Osserman inequality for minimal surfaces in $\mathbb{H}^{n}$. Proc. Amer. Math Soc. 128, 2445-2450 (1999)

[17] Seo, K.: Stable minimal hypersurfaces in the hyperbolic space. J. Korean Math. Soc. 48(2), 253-266 (2011).

[18] Shi, Y., Tian, G.: Rigidity of asymptotically hyperbolic manifolds. Comm. Math. Phys. 259(3), 545-559 (2005).

[19] Tkachev, V.G.: Finiteness of the number of ends of minimal submanifolds in Euclidean space. Manuscripta Math. 82(3-4), 313-330 (1994).

[20] Yau, S.T.: Review of geometry and analysis. Asian J. Math. 4(1), 235-278 (2000). Kodaira's issue 
Department of Mathematics, Universitat Jaume I, Castelló de la Plana, Spain E-mail address: vigigar@postal.uv.es 